\title{
Análise discriminante dos pacientes portadores de glaucoma primário de ângulo aberto através do exame de Doppler colorido e do campo visual
}

\author{
Discriminant analysis of patients with primary open-angle glaucoma by color \\ Doppler exam and visual field
}

Maria Helena Mandello Carvalhães Ramos ${ }^{1}$, Olivier Bergés ${ }^{2}$, João Antonio Prata JR. ${ }^{3}$, Norma Allemann ${ }^{4}$

\section{RESUMO}

Objetivo: Determinar se as variáveis obtidas com exame Doppler colorido associadas às de campo visual são capazes de discriminar olhos normais de olhos glaucomatosos.

Métodos: Foram avaliados prospectivamente 36 pacientes portadores de glaucoma primário de ângulo aberto em uso de medicação antiglaucomatosa (grupo glaucoma) e 20 voluntários normais (grupo controle). Analisou-se a distribuição dos grupos quanto ao sexo, idade, espessura corneana central, pressão intraocular, índices globais da perimetria computadorizada Octopus ("mean defect" e "loss variance") e os parâmetros do Doppler colorido (velocidade sistólica máxima e índice de resistência).

Resultados: Não houve diferença estatística significativa entre os grupos em relação à idade, espessura corneana e pressão intraocular. A análise discriminante mostrou que as variáveis: índice de resistência (IR) da artéria ciliar curta posterior (ACCP), "mean defect" (MD) e "loss variance" (LV) apresentaram influência estatisticamente significativa para o diagnóstico positivo de glaucoma. A função discriminante obtida foi: $-3,637+0,109$ MD + 0,028 LV + 4,325 IR ACCP. A probabilidade do diagnóstico positivo do glaucoma a partir do "score" -1,61 foi de 90\%.

Conclusão: Foi possível identificar através da análise discriminante, quais das variáveis do Doppler colorido que, associados às do campo visual, permitiram diferenciar pacientes glaucomatosos de normais. Essas variáveis foram: índice de resistência da artéria ciliar curta posterior e índices da perimetria computadorizada "mean defect" e "loss variance".

Descritores: Glaucoma de ângulo aberto; Ultrassonografia doppler em cores/métodos; Pressão intraocular; Velocidade do fluxo sanguíneo/fisiologia

\begin{abstract}
Purpose: To determine if variables from color Doppler and visual field exam could discriminate normal from glaucomatous eyes.

Methods: Prospectively, 36 patients with primary open-angle glaucoma (glaucoma group) and 20 normal volunteers (control group) were studied. Gender, age, central corneal thickness, intraocular pressure, Octopus automated perimetry global indices (mean defect and loss variance) and several parameters of the color Doppler (peak systolic velocity and resistivity index) were compared between groups.

Results: There was no statistically significant difference in age, central corneal thickness and intraocular pressure. Discriminant analysis showed that the variables: resistivity index (RI) in the short posterior ciliary artery (SPCA), mean defect (MD) and loss variance (LV) had presented significant influence for the positive diagnosis of glaucoma. The gotten discriminant function was: $-3.637+0.109 \times M D+0.028 \times L V+4.325 \times R I S P C A$. Considering score -1.61 , the probability of positive diagnosis of glaucoma was $90 \%$. Conclusion: Through discriminant analysis it was possible to identify which of the color Doppler variables that associated to the visual field variables allowed differentiate normal from glaucomatous patients. These variables were: resistivity index in the short posterior ciliary artery, and the visual field variables, mean defect and loss variance.
\end{abstract}

Keywords: Glaucoma, open-angle; Ultrasonography, doppler, color/methods; Intraocular pressure; Blood flow velocity/physiology

\section{INTRODUÇÃO}

O diagnóstico do glaucoma baseia-se na história clínica, tonometria (medida da pressão intraocular), gonioscopia, avaliação da cabeça do nervo óptico, avaliação da camada de fibras nervosas da retina e avaliação do campo visual ${ }^{(1,2)}$. Entretanto, algumas vezes, esta propedêutica não permite a diferenciação entre suspeitos e glaucomatosos. Desta forma, diversas propedêuticas e estratégias têm sido desenvolvidas no intuito de permitir o diagnóstico mais preciso e precoce do glaucoma. Dentre elas, destacam-se modernas técnicas para avaliação anatômica ou funcional das estruturas envolvidas no dano glaucomatoso(3-6).
Dentre as novas propedêuticas para avaliação funcional, especial atenção recai sobre o Doppler colorido que permite a avaliação da circulação sanguínea retrobulbar. O Doppler colorido é um método de imagem não invasivo que associa a ecografia modo B em tempo real à técnica Doppler, permitindo assim a análise das características do fluxo sanguíneo dos vasos retrobulbares ${ }^{(7-12)}$.

Birinci et al. ${ }^{(11)}$ e Rankin et al. ${ }^{(13)}$ estudaram pacientes com glaucoma primário de ângulo aberto (GPAA) com pressão intraocular (PIO) controlada (PIO <21 mmHg) com medicação tópica e encontraram uma redução da velocidade sistólica máxima (VSM) e velocidade diastólica final (VDF) e um aumento do índice de resistência (IR) da (Paris - França) e Departamento de Oftalmologia da Universidade Federal de São Paulo - UNIFESP.

Physician, Departamento de Oftalmologia, Universidade Federal de São Paulo - UNIFESP - São Paulo (SP), Brazil.

2 Physcian, Le Service d'Imagerie, Fondation Ophtalmologique Adolphe Rothschild, Paris, France.

3 Physician, Departamento de Oftalmologia, Faculdade de Medicina, Universidade Federal do Triângulo Mineiro, Uberaba (MG), Brazil.

${ }^{4}$ Physician, Departamento de Oftalmologia, Universidade Federal de São Paulo - UNIFESP - São Paulo (SP), Brazil.

Funding: No specific financial support was available for this study.

Disclosure of potential conflicts of interest: M.H.M.C.Ramos, None; O.Bergés, None; J.A. Prata Jr, None; N.Allemann, None.

Correspondence address: Norma Allemann. Av. Indianópolis, 1797 - São Paulo (SP) - 04063-003 Brazil - E-mail: norma.allemann@pobox.com

Editorial Note: After completing the confidential analysis of the manuscript, ABO discloses, with her agreement, the name Dr. Ruth Rosenhek Schor as a reviewer. We thank her effort and expertise in participating in this process. 
artéria central da retina (ACR) e das artérias ciliares curtas posteriores (ACCP) em comparação com o grupo controle.

Akarsu \& Bilgili(14) encontraram uma diminuição da VSM e da VDF e aumento do IR na ACR, na artéria oftálmica (AO) e na ACCP em pacientes com GPAA. Neste estudo, os pacientes portadores de hipertensão ocular apresentaram diferença estatística significativa apenas no IR da ACCP em relação ao grupo controle.

$\mathrm{Na}$ literatura, alterações dos vasos retrobulbares encontradas através do Doppler têm sido correlacionadas com o campo visual, porém até o momento nenhum estudo foi realizado procurando estabelecer o diagnóstico do glaucoma utilizando os índices globais da perimetria computadorizada com os parâmetros obtidos através do Doppler colorido.

O objetivo deste estudo é identificar quais das variáveis obtidas com exame Doppler colorido que, associadas às de campo visual, possam permitir discriminar olhos normais de glaucomatosos.

\section{MÉTODOS}

Foi realizado a estratégia de pesquisa estudo de caso de 36 pacientes portadores de glaucoma primário de ângulo aberto em uso de medicação antiglaucomatosa (grupo glaucoma) e 20 voluntários normais (grupo controle) no período de maio de 2002 a maio de 2003.

Definiu-se como paciente portador de glaucoma primário de ângulo aberto, aquele que apresentava as seguintes características: presença de alteração do nervo óptico (NO) e alteração de campo visual (CV) típicas de glaucoma, pressão intraocular maior que $21 \mathrm{mmHg}$ sem uso de medicação e ângulo da câmara anterior aberto com aparência normal.

A alteração do NO foi caracterizada pela presença de pelo menos 2 dos seguintes critérios: E/D maior que 0,6 em seu maior eixo, perda localizada da rima neural ("notch"), hemorragia do disco óptico ou assimetria de escavação $>0,3$.

Definiu-se como defeito de CV glaucomatoso os seguintes critérios: 3 ou mais pontos adjacentes em um mesmo hemisfério com perda maior ou igual a 5 decibéis $(d B)$, sendo que em 1 destes pontos a perda era maior ou igual a $10 \mathrm{~dB}$ ou 3 ou mais pontos adjacentes no meridiano horizontal nasal apresentando diferença de $5 \mathrm{~dB}$ ou mais do mesmo grupo de pontos no hemisfério oposto. Todos os campos analisados apresentavam índices de confiabilidade aceitáveis. No grupo de portadores de GPAA foram incluídos todos os tipos de defeito campimétrico: inicial, moderado ou avançado.

Critérios de exclusão dos pacientes: uveíte, cirurgia intraocular prévia, hipertensão arterial, diabetes, síndrome de Raynaud, arterite de células gigantes ou enxaqueca.

Todos os pacientes portadores de GPAA que participaram desta pesquisa estavam em uso de medicação antiglaucomatosa (maleato de timolol a 0,5\% e/ou tartarato de brimonidina a 0,2\%).

Para o grupo controle foram selecionados pacientes hígidos que apresentavam os seguintes critérios: PIO entre 10 e 20 mmHg; E/D menor que 0,5, CV dentro dos padrões da normalidade e exame fundoscópico e à lâmpada de fenda compatível com a normalidade.

Em todos os grupos, apenas um olho de cada paciente foi escolhido de forma aleatória. Todos os pacientes foram submetidos a exame de acuidade visual, tonometria, oftalmoscopia, paquimetria ultrassônica, perimetria computadorizada (Perímetro Octopus 1-2-3, estratégia dinâmica e programa G1) e análise do fluxo sanguíneo dos vasos retrobulbares através do aparelho Doppler colorido
(Philips HDI - Scientific Meical Systems, Bothell, EUA com sonda linear de 5-12 MHz). O intervalo de tempo máximo entre o exame de perimetria computadorizada e de Doppler colorido foi de 7 dias. Analisou-se a distribuição dos grupos quanto ao sexo, idade, espessura corneana central, pressão intraocular (PIO), índices globais da perimetria computadorizada ("mean defect" e "loss variance") e os diversos parâmetros do Doppler colorido (velocidade sistólica máxima da artéria central da retina, índice de resistência da artéria central da retina e artéria ciliar curta posterior) (Figuras 1-4).

Foram aplicados os testes $t$ de Student para estudo das variáveis quantitativas entre os grupos e o teste exato de Fisher para o estudo das variáveis qualitativas. Para as variáveis que tinham informações que diferenciavam os grupos controle e glaucoma, empregou-se a análise discriminante.

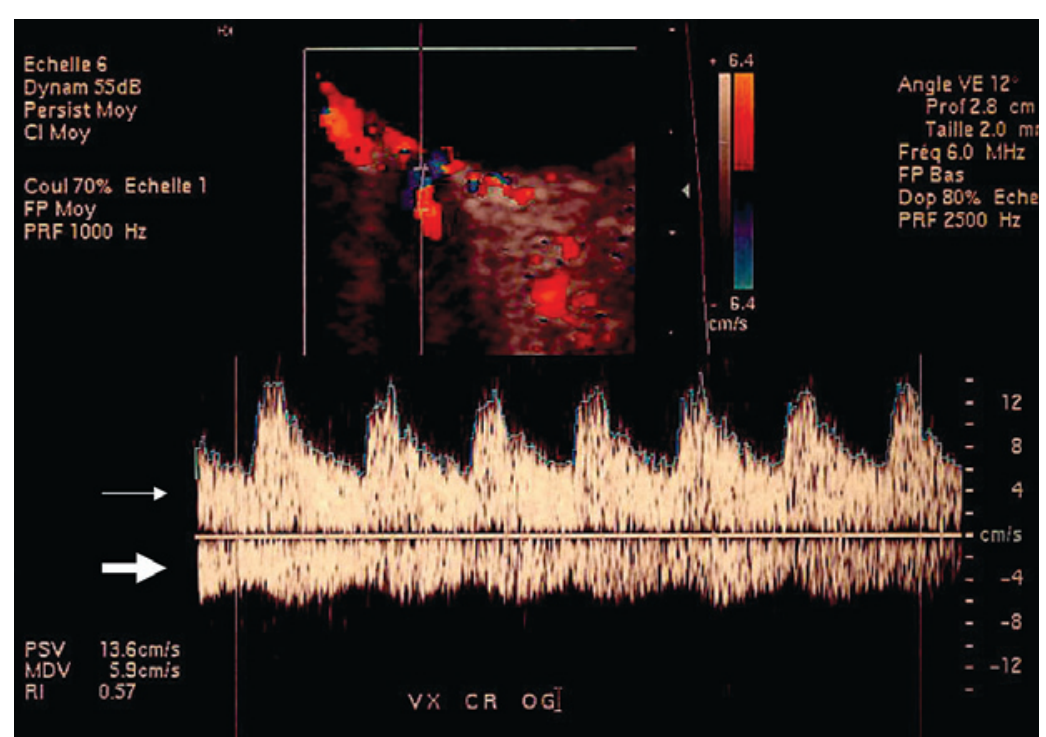

Figura 1. Registro doppler velocimétrico do complexo artéria/veia central da retina de um índivíduo normal. Observa-se um componente arterial (seta fina) pulsátil acima da linha horizontal e o componente venoso (seta) laminar, abaixo da linha horizontal. Controle 28.

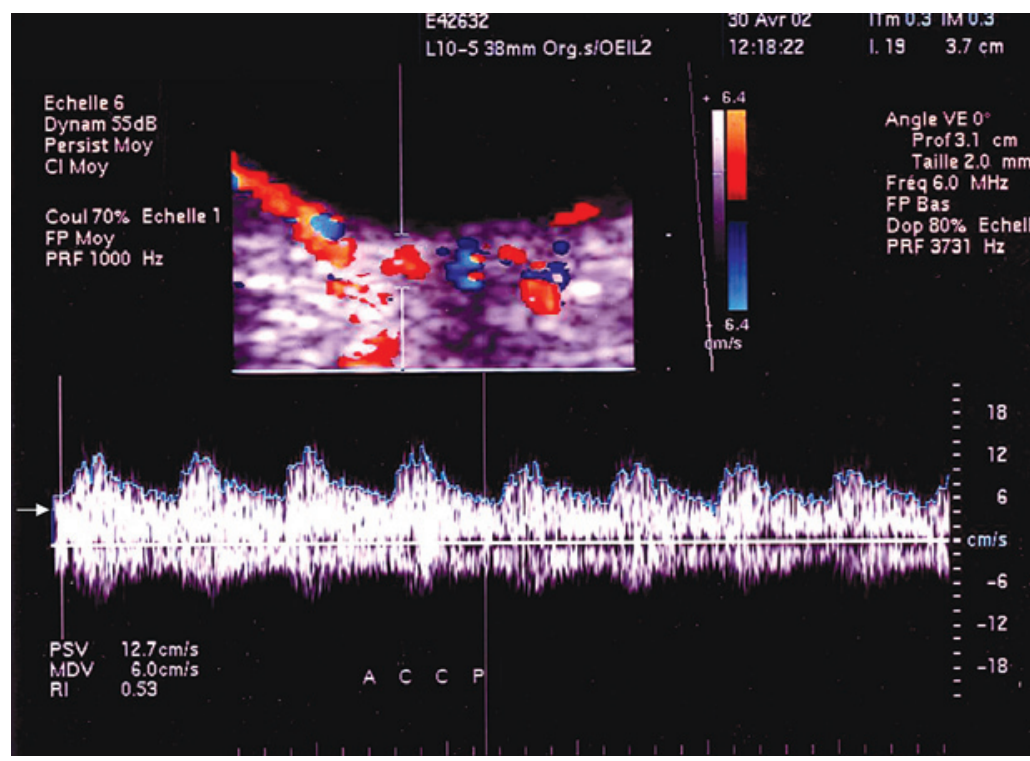

Figura 2. Registro doppler velocimétrico da artéria ciliar curta posterior de um indivíduo normal. A análise espectral desta artéria fornece um componente exclusivamente positivo (seta). Controle 28. 


\section{RESULTADOS}

Não houve diferença estatística significante entre os grupos em relação à idade $(p=0,155)$, a espessura corneana central $(p=0,147)$ e a pressão intraocular $(p=0,112)$. A média de idade do grupo de pacientes com GPAA foi de 62,9 $\pm 7,1$ anos e do grupo controle foi de 60,6 $\pm 6,9$ anos. As médias da PIO foram 17,2 $\pm 5,1 \mathrm{mmHg}$ nos olhos dos pacientes do grupo glaucoma e 15,5 $\pm 2,6 \mathrm{mmHg}$ no grupo controle.

Em relação aos parâmetros do campo visual o "mean defect" (MD) médio do grupo controle foi de $-0,6 \pm 1,1 \mathrm{~dB}$ e de $14,2 \pm 8,1 \mathrm{~dB}$ no grupo glaucoma ( $p=0,001)$. O "loss variance" (LV) médio do grupo controle foi de $2 \pm 1,1 \mathrm{~dB}$ e 23,8 $\pm 24,4 \mathrm{~dB}$ no grupo glaucoma $(p=0,001)$.

Considerando-se os parâmetros provenientes do exame de Doppler colorido, a média da velocidade sistólica máxima (VSM) da artéria central da retina (ACR) analisada através do Doppler colorido foi 10,6 $\pm 3,3 \mathrm{~cm} / \mathrm{s}$ no grupo glaucoma (Figura 1) e 12,8 $\pm 2,8 \mathrm{~cm} / \mathrm{s}$ no grupo controle $(p=0,014)$ (Gráfico 1).

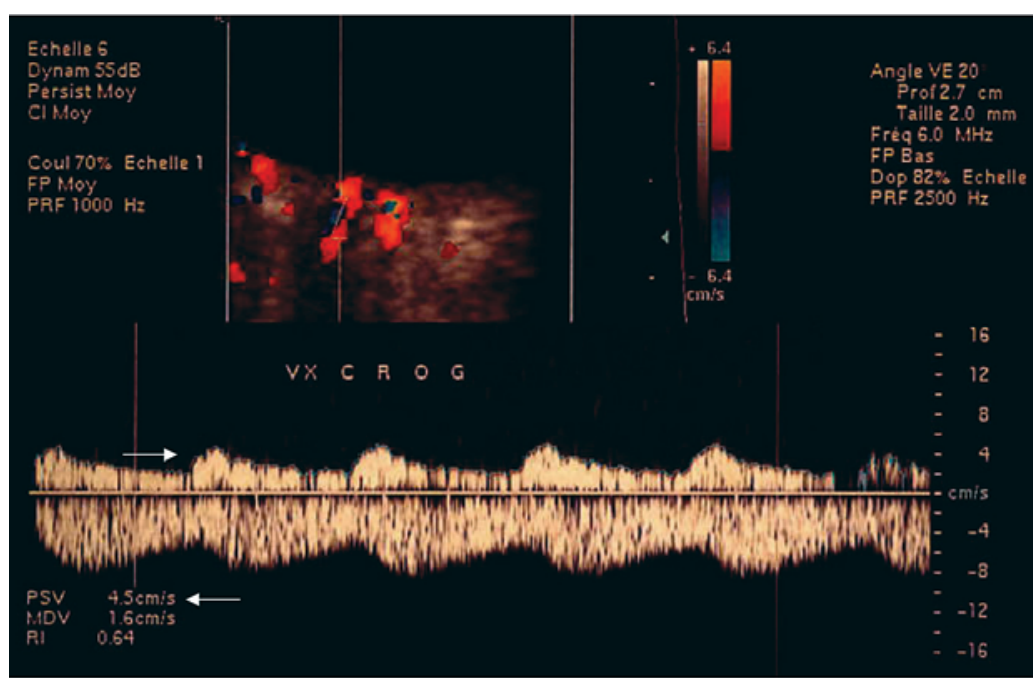

Figura 3. Registro doppler velocimétrico do complexo artéria/veia central da retina do paciente 7 portador de GPAA. Observa-se, na análise espectral, uma diminuição da velocidade sistólica máxima (seta).

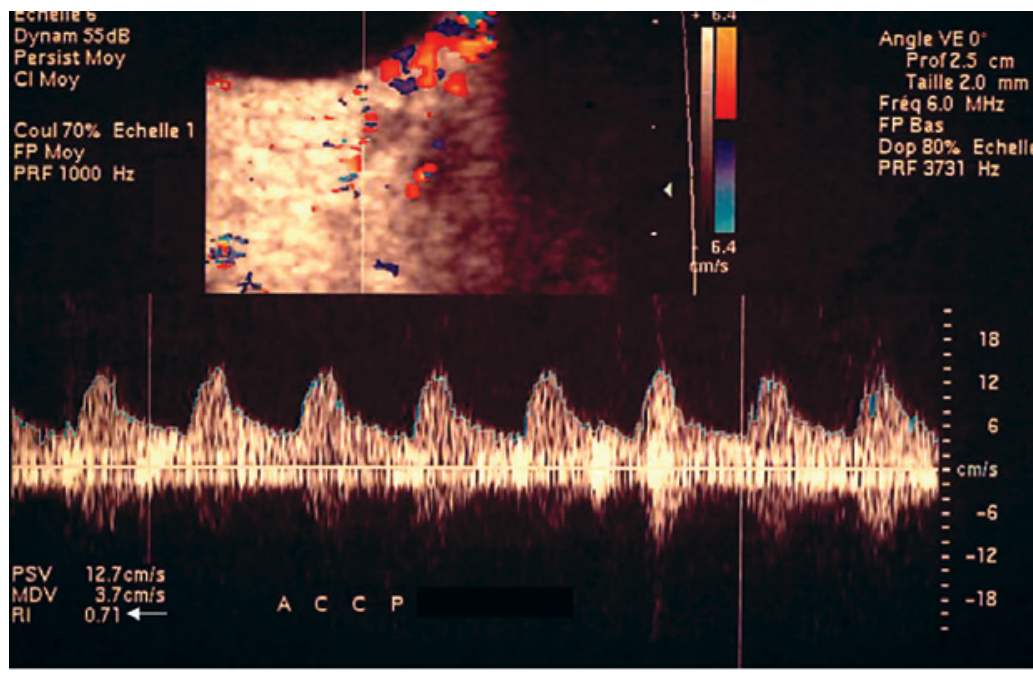

Figura 4. Registro doppler velocimétrico da artéria ciliar curta posterior de um paciente portador de GPAA. Observa-se na análise espectral um aumento do índice de resistência (seta). Paciente 56.
A média do índice de resistência (IR) da ACR do grupo glaucoma foi de 0,75 $\pm 0,1$ e do grupo controle foi de 0,63 $\pm 0,03$ $(p=0,01)$ (Gráfico 2).

As médias do IR da artéria ciliar curta posterior (ACCP) no grupo glaucoma e no grupo controle foram, respectivamente, 0,67 \pm $0,1$ e 0,54 $\pm 0,03$ ( $p=0,001)$ (Gráfico 3).

A análise discriminante mostrou que as variáveis: IR da ACCP MD e LV apresentaram influência estatisticamente significante para o diagnóstico positivo de glaucoma. A função discriminante obtida foi: $-3,637+0,109 \mathrm{MD}+0,028 \mathrm{LV}+4,325$ IR ACCP. A partir do "score" -1,61 a probabilidade do diagnóstico positivo do glaucoma foi de $90 \%$.

\section{DISCUSSÃO}

Na literatura, alterações dos vasos retrobulbares encontradas através do método de exame Doppler colorido têm sido correlacionadas com o campo visual, porém até o momento nenhum estudo foi realizado procurando estabelecer o diagnóstico do glaucoma utilizando esses parâmetros da forma que foi empregada neste estudo. A análise discriminante mostrou que as variáveis: índice de resistência (IR) da artéria ciliar curta posterior (ACCP), "mean defect" (MD) e "loss variance" (LV) apresentam influência estatisticamente significante para o diagnóstico positivo de glaucoma. A equação discriminante $(-3,637+0,109 \mathrm{MD}+0,028 \mathrm{LV}$ $+4,325$ IR ACCP) obtida apresentou, a partir do "score" $-1,61$, uma probabilidade de diagnóstico positivo do glaucoma de $90 \%$.

Guthoff et al. ${ }^{(15)}$ mostraram, através do aumento agudo da PIO pelo método de sucção escleral, uma relação positiva entre a PIO e o IR da ACR. Joos et al. ${ }^{(16)}$ demostraram, em sua pesquisa com aumento artificial da PIO em voluntários normais, que as características hemodinâmicas da artéria ciliar curta posterior são dependentes da $\mathrm{PIO}$, ou seja, observaram um aumento do IR e uma diminuição da VSM e da VDF com aumento da PIO.

Harris et al. ${ }^{(17)}$ estudaram a circulação retrobulbar através do Doppler colorido de 125 voluntários sem alteração ocular, com idade entre 20 e 90 anos e encontraram aumento do índice de resistência da artéria ciliar curta posterior em pacientes do sexo feminino em relação à idade.

Doughty \& Zaman ${ }^{(18)}$, através de um estudo de metaanálise, demonstraram que $10 \%$ de diferença na espessura corneana é capaz de alterar a $\mathrm{PIO}$ em aproximadamente $3,5 \mathrm{mmHg}$.

Silva et al.(19), estudaram a circulação retrobulbar através do Doppler colorido de pacientes com glaucoma em uso de tartarato de brimonidina a 0,2\% e encontraram um aumento da VSM e VDF na artéria oftálmica do olho direito e redução do $I R$ da $A O$ do olho esquerdo. Nos demais vasos estudados não foi encontrada alteração significante.

No presente estudo, não foram encontradas diferenças estatisticamente significante entre os grupos quanto às variáveis com possível influência sobre o fluxo sanguíneo ocular (PIO, paquimetria, idade), o que reforça a homogeneidade dos grupos.

Cellini et al. ${ }^{(20)}$ dividiram os pacientes portadores de GPAA com PIO controlada com medicação antiglaucomatosa em dois grupos de acordo com as alterações dos índices globais: grupo A (menos alterado) e grupo B (mais alterado). Os pacientes do grupo B apresentaram alterações mais importantes do índice de resistência da artéria ciliar curta posterior em comparação com o grupo A.

Liu et al.(21) estudaram 25 pacientes portadores de GPAA com PIO controlada ( $\mathrm{PIO}<22 \mathrm{mmHg}$ ) com medica- 


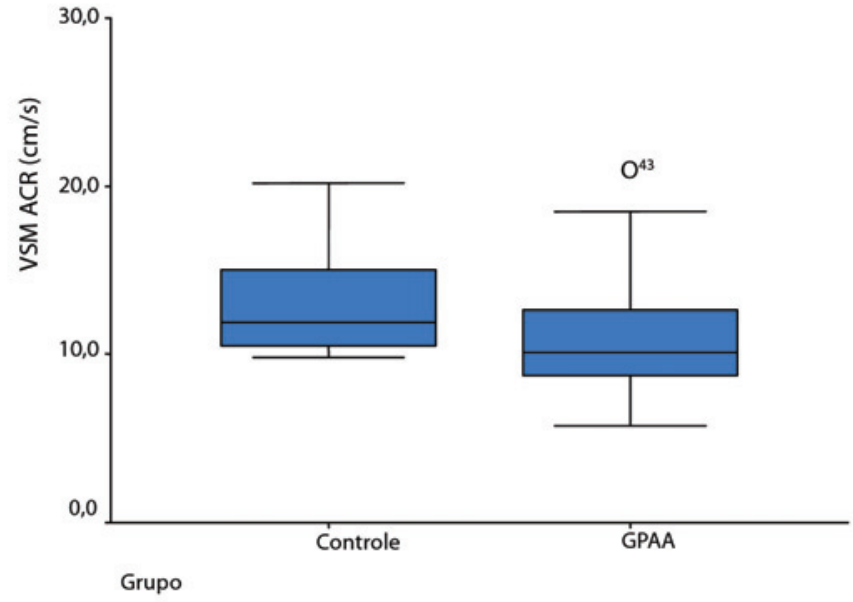

Gráfico 1.Gráfico de distribuição da velocidade sistólica máxima (VSM) (cm/s) da artéria central da retina (ACR), no grupo de pacientes portadores de glaucoma primário de ângulo aberto (GPAA) e no grupo controle. As barras horizontais externas representam os valores mínimo e máximo; as barras horizontais delimitam a caixa, o $1^{\circ}$ e $3^{\circ}$ quartil; $\mathrm{e}$ a barra horizontal no interior da caixa representa a mediana.

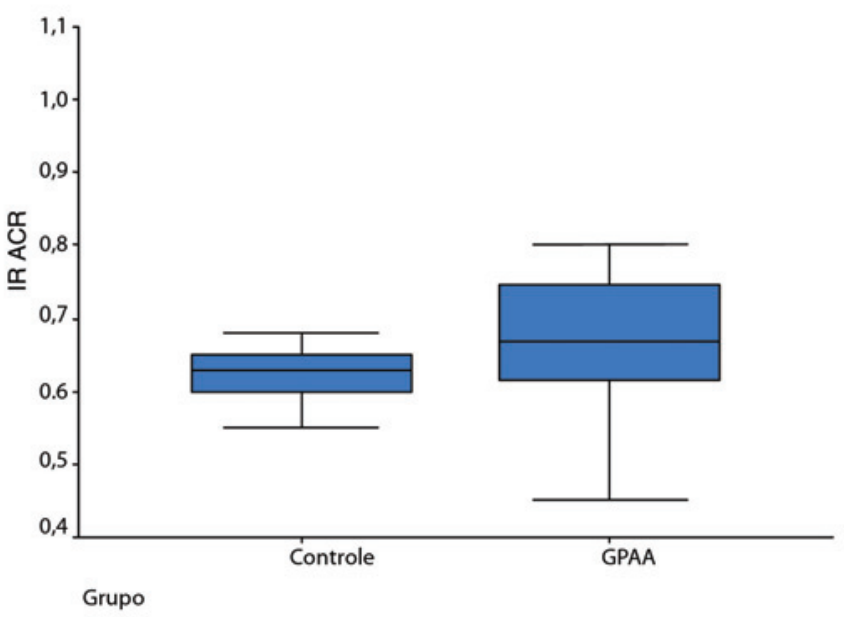

Gráfico 2. Gráfico de distribuição do índice de resistência (IR) da artéria central da retina $(A C R)$, no grupo de pacientes portadores de glaucoma primário de ângulo aberto (GPAA) e no grupo controle. As barras horizontais externas representam os valores mínimo e máximo; as barras horizontais delimitam a caixa, o $1^{\circ}$ e $3^{\circ}$ quartil; e a barra horizontal no interior da caixa representa a mediana.

ção antiglaucomatosa e classificaram esses pacientes como portadores de glaucoma inicial e avançado conforme as alterações dos índices globais apresentados no campo visual. Os pacientes portadores de glaucoma inicial apresentaram diminuição da velocidade sistólica máxima apenas na artéria ciliar curta posterior. Os pacientes portadores de glaucoma avançado apresentaram diminuição da velocidade sistólica máxima e da velocidade diastólica final e aumento do índice de resistência tanto na artéria central da retina quanto na artéria ciliar curta posterior.

No presente estudo, foi encontrado, mesmo com o controle da $\mathrm{PIO}$, diferença estatística dos parâmetros do fluxo sanguíneo da artéria central da retina e da artéria ciliar curta posterior medidos através do Doppler colorido entre os grupos controle e glaucoma, sendo este resultado concordante com os estudos apresentados por Birinci et al. ${ }^{(11)}$, Rankin et al. ${ }^{(13)}$ e Akarsu \& Bilgili(14).

\section{CONCLUSÃO}

No presente estudo, foi possível identificar, através da análise discriminante, quais das variáveis obtidas através do Doppler colo-

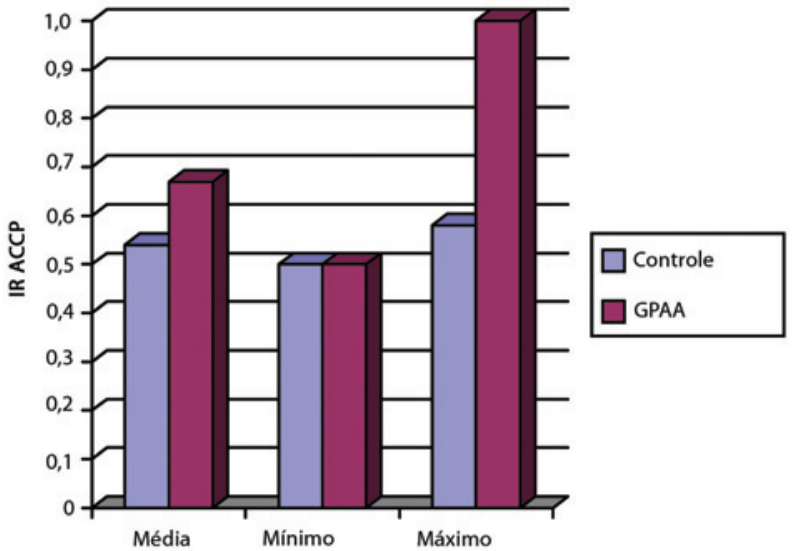

Gráfico 3. Gráfico de distribuição do índice de resistência (IR) da artéria ciliar curta posterior (ACCP), no grupo de pacientes portadores de glaucoma primário de ângulo aberto (GPAA) e no grupo controle. As barras horizontais representam os valores da mediana, mínimo e máximo; as barras verticais representam os valores do índice de resistência.

rido (índice de resistência da artéria ciliar curta posterior) que, associadas às variáveis do campo visual ("mean deviation" e "loss variance"), permitiram discriminar pacientes glaucomatosos de normais, independentemente do estabelecimento do controle medicamentoso da pressão ocular.

\section{REFERÊNCIAS}

1. Tielsch JM, Katz J, Singh K, Quigley HA, Gottsch JD, Javitt J, Sommer A. A population-based evaluation of glaucoma screening: the Baltimore Eye Survey. Am J Epidemiol. 1991;134(10): 1102-10

2. Teoh SL, Allan D, Dutton GN, Fouds WS. Brightness discrimination and contrast sensibility in chronic glaucoma - a clinical study. Br J Ophthalmol. 1990;74(4):215-9.

3. Weinreb RN, Zangwill L, Berry CC, Bathija R, Sample PA. Detection of glaucoma with scanning laser polarimetry. Arch Ophthalmol. 1998;116(12):1583-9.

4. lester M, Mikelberg FS, Courtright P, Drance SM. Correlation between the visual field indices and Heidelberg retina tomograph parameters. J Glaucoma. 1997;6(2):78-82.

5. Flammer J, Orgül S. Optic nerve blood-flow abnormalities in glaucoma. Prog Retin Eye Res. 1998;17(2):267-89.

6. Harris A, Kagemann L, Cioff GA. Assessment of human ocular hemodynamics. Surv Ophthalmol. 1998:42(6):509-33.

7. Aburn NS, Sergott RC. Orbital colour Doppler imaging. Eye. 1993;7(Pt 5):639-47.

8. Sergott RC, Aburn NS, Trible JR, Costa VP, Lieb WE Jr, Flaharty PM. Color Doppler imaging: methodology and preliminary results in glaucoma. Surv Ophthalmol. 1994;38 Suppl 1: S65-70; discussion S70-1. Review. Erratum in: Surv Ophthalmol. 1994;39(2):165.

9. Lachkar Y, Migdal C, Dhanjil S. Effect of brimonidine tartrate on ocular hemodynamic measurements. Arch Ophthalmol. 1998;116(12):1591-4.

10. Harris A, Spaeth GL, Sergott RC, Katz LJ, Cantor LB, Martin BJ. Retrobulbar arterial hemodynamic effects of betaxolol and timolol in normal-tension glaucoma. Am J Ophthalmol. 1995;120(2):168-75.

11. Birinci $H$, Danaci M, Oge I, Erkan ND. Ocular blood flow in healthy and primary open-angle glaucomatous eyes. Ophthalmologica. 2002;216(6):434-7.

12. Costa VP. O uso do Color Doppler em oftalmologia. Arq Bras Oftalmol. 1994;57(3):150-3.

13. Rankin SJ, Walman BE, Buckley AR, Drance SM. Color Doppler imaging and spectral analysis of the optic nerve vasculature in glaucoma. Am J Ophthalmol. 1995;119(6):685-93.

14. Akarsu C, Bilgili MY. Color Doppler imaging in ocular hypertension and open-angle glaucoma. Graefes Arch Clin Exp Ophthalmol. 2004;242(2):125-9.

15. GuthoffRF, Berger RW, Winkler P, Helmke K, Chumbley LC. Doppler ultrasonography of the ophthalmic and central retinal vessels. Arch Ophthalmol. 1991;109(4):532-6.

16. Joos KM, Kay MD, Pillunat LE, Harris A, Gendron K, Feuer WJ, Steinwand BE. Effect of acute intraocular pressure changes on short posterior ciliary artery haemodynamics. Br J Ophthalmol. 1999;83(1):33-8.

17. Harris A, Harris M, Biller J, Garzozi H, Zarfty D, Ciulla TA, Martin B. Aging effects the retrobulbar circulation differently in women and men. Arch Ophthalmol. 2000;1 18(8):1076-80.

18. Doughty MJ, Zaman ML. Human corneal thickness and its impact on intraocular pressure measures: a review and meta-analysis approach. Surv Ophthalmol. 2000;44(5):367-408.

19. Silva MRBM, Rollo HA, Schellini SA. Efeito da brimonidina na circulação retrobulbar em pacientes com glaucoma: estudo com doppler colorido. Arq Bras Oftalmol 2001:64(4):309-13.

20. Cellini M, Possati GL, Sbrocca M, Caramazza N. Correlation between visual field and color Doppler parameters in chronic open angle glaucoma. Int Ophthalmol. 1996-1997;20(4):215-9.

21. Liu CJ, Chiou HJ, Chiang SC, Chou JC, Chou YH, Liu JH. Variations in ocular hemodynamics in patients with early and late glaucoma. Acta Ophthalmol Scand. 1999;77(6):658-62. 